\title{
Using Performance in Multiple Simulated Scenarios to Assess Bronchoscopy Skills
}

\author{
Lars Konge Henrik Arendrup Christian von Buchwald Charlotte Ringsted \\ Centre for Clinical Education, University of Copenhagen and Capital Region of Denmark, Copenhagen, Denmark
}

\section{Key Words}

Assessment method · Bronchoscopy · Education •

Procedural training $\cdot$ Simulation $\cdot$ Virtual reality

\begin{abstract}
Background: International guidelines suggest that trainees should perform at least 100 flexible bronchoscopies in a supervised setting, but this number is not evidence based. An objective assessment method could provide educational feedback to trainees and help supervisors decide when basic competency is established. No former assessment instrument has been able to distinguish between trainees and experts. Objectives: The aim of this study was to explore the validity and reliability of a new assessment procedure relating to testing operators across multiple tasks with increasing difficulty using a standardized scoring form. Methods: The test was administered on a virtual reality bronchoscopy simulator to a total of 42 test subjects (14 senior consultants, 14 trainees and 14 medical students). The inter-rater reliability of the test procedure was explored according to examination of test results from 10 subjects using 3 raters ( 2 blinded). Results: We found a high inter-rater reliability (Cronbach's $\alpha=0.90$ ). The assessment procedure sufficiently differentiated the performance of the 3 groups ( $p<0.001$ ). Assessment of 6 procedures was necessary to secure a generalizability coefficient $>0.80$. Conclusions: The new assessment procedure proved both valid and reliable.
\end{abstract}

Copyright $\odot 2011$ S. Karger AG, Basel
(C) 2011 S. Karger AG, Basel

0025-7931/11/0816-0483\$38.00/0

Fax +4161306 1234

E-Mail karger@karger.ch

www.karger.com
Accessible online at: www.karger.com/res
For editorial comment see p. 446

\section{Introduction}

Flexible bronchoscopy is usually an extraordinarily safe procedure, and major complications such as bleeding, respiratory depression, cardiorespiratory arrest, arrhythmia and pneumothorax occur in $<1 \%$ of cases [1]. Still, sufficiently trained personnel is of paramount importance to maximize patient comfort, safety and yield. Patients undergoing bronchoscopy performed by novice bronchoscopists have an increased complication rate during the first trimester of bronchoscopists' training [2]. Moreover, operator experience influences the success rate of positive biopsy material from a visible tumor [3] and the performance of transbronchial needle aspiration [4]. Bronchoscopy guidelines from the US and the UK [1, 5] suggest that trainees should perform at least $100 \mathrm{flex}$ ible bronchoscopies in a supervised setting to establish basic competency. This number is not evidence based and there is no uniform agreement between bronchoscopists [6]. The controversy regarding the issue of requiring a minimum number of procedures relates to the recognition that the manual dexterity and confidence of trainees is highly variable [7]. Therefore, an objective assessment method could help supervisors decide when basic competency is established $[8,9]$. However, objective assessment of bronchoscopy skills is not an easy task. Traditional testing of knowledge is insufficient as there is no relationship between theoretical knowledge and techni- 
cal skills, self-assessed practical expertise or number of bronchoscopies performed $[10,11]$. Hence, there is a need for performance-based assessment. Prior reports on evaluations of trainees' performances are based on either the opinion of a single thoracic surgeon [12] or of an experienced bronchoscopy nurse [13]. However, this approach is rather subjective, using nonvalidated methods. Virtual reality bronchoscopy simulators have been proposed as ideal 'assessors' of bronchoscopy skills as they provide unbiased parameters of performance (i.e. procedure time, percentage of segments visualized and number of collisions). Indeed, one simulator study showed that skilled physicians performed bronchoscopies faster, more thoroughly and with fewer collisions than complete novices [13], but unfortunately, these simulator-provided metrics could not differentiate between performance of intermediates and experts. Another study showed no differences between experts and novices that had tried the simulator 5 times [14], indicating a significant ceiling effect of the metrics. Therefore, we cannot base an assessment solely on the parameters provided by the simulator. Recently, two measurement instruments were created to assess bronchoscopy skills and were tested using 2 highly skilled bronchoscopy experts as raters [15]. The study found a high inter- and intra-rater agreement, and both measurement instruments were able to distinguish complete novices from experienced bronchoscopists. However, there were no significant statistical differences in the performance of the trainees compared with intermediate experience and experts.

The poor discriminative ability of prior assessment methods might relate to several factors including testing with too easy tasks, testing with too few procedures, and finally, the lack of performance indicators of clinical relevance to supplement the outcome parameters provided by the simulator metrics.

As an attempt to solve the problems of lack of discriminative ability, we developed a new assessment approach. This included 6 scenarios where the participants' ability to find tumors, describe their localization and perform different tasks were tested. Tasks with increasing difficulty were included to avoid a ceiling effect and favor the good bronchoscopist, as the ability to transfer skills to a new situation is one of the trademarks of experts [16]. To minimize subjectivity, we used a checklist with items that could be rated by watching blinded video recordings of the procedures.

The purpose of this study was to investigate the reliability and validity of this assessment approach. In short, the new approach should precisely and consistently be able to distinguish bronchoscopy experts from the less experienced. In addition, the assessment should not take unnecessarily long time, which is why we also wanted to investigate the optimal balance between time consumption and precision.

\section{Materials and Methods}

\section{Development of the Assessment Tool}

The assessement tool was developed by a team of 5 physicians: 3 clinical experts of bronchoscopy (Nils Milman - pulmonary medicine, H.A. - thoracic surgery, and C.v.B. - otorhinolaryngology), 1 expert in educational measurement (C.R.) and 1 experienced bronchoscopist with expert knowledge of a virtual reality bronchoscopy simulator (L.K.).

To provide a standardized environment where test persons could safely perform bronchoscopies without intervention from a supervisor, the assessment tool was designed to be used on a simulator (Endoscopy AccuTouch System with bronchoscopy modules from Immersion Medical, Gaithersburg, Md., USA). It consists of a computer, an interface for the flexible tube, a proxy bronchoscope and software modules. When the proxy bronchoscope is inserted into the interface, the software creates a realistic image of the virtual patient's airways. When the scope is moved around, the image on the monitor changes accordingly, and the interface adds appropriate resistance depending on the position in the bronchial tree. Local anesthesia and saline are administered via a foot pedal, and a plastic cord can be inserted into the working channel of the scope to simulate forceps, brush or needle. The simulator provides different feedback, i.e. time of procedure, percent of bronchial segments entered and number of scope collisions with airway walls.

To supplement these simulator metrics, we created a clinically meaningful checklist. It consists of 28 items that can be scored dichotomously. The first draft was carefully revised before and after a pilot study was done. Finally, we decided to test the tool shown in table 1 on a larger scale (see below).

\section{The Validation Process}

In order to examine the reliability, validity and the optimal duration of the test procedure, the test was performed by a total of 42 persons representing 3 groups: medical students with no prior experience in bronchoscopy, junior consultants who had each performed $>10$ but $<100$ bronchoscopies (and therefore still trainees according to international guidelines), and senior consultants who had each performed more than 500 bronchoscopies. Before the test, the medical students were shown a bronchoscopy instruction video by E. James Britt, MD, but apart from this, they were assessed in the same way as the other participants. None of the test subjects had tried the simulator prior to the test, but each was allowed a total of 30 min of 'warm-up' to get accustomed to the simulator.

Each person tested had to perform 6 procedures: first, 2 diagnostic bronchoscopies where the test subject had to perform a thorough bronchoscopy, find an unknown number of hidden tumors and state the localization of each tumor. Next, the participant had to perform 2 bronchialveolar lavages (BALs); lavage with $50 \mathrm{ml}$ of lukewarm saline followed by suction had to be repeated 3 times in either the lateral or medial segment of the middle lobe 
Table 1. The assessment tool

\begin{tabular}{|c|c|c|c|c|}
\hline \multicolumn{5}{|c|}{ General warm-up: module 1, case 3 (max. 15 min) } \\
\hline & & & Score & Time \\
\hline \multirow{7}{*}{$\begin{array}{l}\text { Module 1: } \\
\text { Case } 1 \\
\max .15 \mathrm{~min}\end{array}$} & \multirow[t]{2}{*}{ Tumor 1} & \multicolumn{2}{|l|}{ Tumor photographed? } & \\
\hline & & $\begin{aligned} \text { Correct localization? } & \text { Right: middle lobe bronchus } \\
& \text { Right: bronchus to segment } 4 \\
& \text { Right: bronchus to lateral part of middle lobe }\end{aligned}$ & & \\
\hline & \multirow[t]{2}{*}{ Tumor 2} & Tumor photographed? & & \\
\hline & & $\begin{array}{ll}\text { Correct localization? } & \text { Left: bronchus to lingua (upper lobe bronchus accepted) } \\
& \text { Left: bronchus to segments } 4 \text { and } 5\end{array}$ & & \\
\hline & \multirow[t]{2}{*}{ Tumor 3} & Tumor photographed? & & \\
\hline & & $\begin{array}{ll}\text { Correct localization? } & \text { Left: bronchus to apical part of lower lobe } \\
& \text { Left: bronchus to segment } 6\end{array}$ & & \\
\hline & \multicolumn{3}{|c|}{ Time $\rightarrow \rightarrow \rightarrow \rightarrow \rightarrow \rightarrow \rightarrow \rightarrow \rightarrow \rightarrow \rightarrow \rightarrow \rightarrow \rightarrow \rightarrow \rightarrow \rightarrow \rightarrow \rightarrow \rightarrow \rightarrow \rightarrow \rightarrow \rightarrow \rightarrow \rightarrow \rightarrow \rightarrow \rightarrow \rightarrow \rightarrow \rightarrow \rightarrow \rightarrow \rightarrow \rightarrow \rightarrow \rightarrow \rightarrow \rightarrow \rightarrow \rightarrow \rightarrow$} & \\
\hline \multirow{5}{*}{$\begin{array}{l}\text { Module 1: } \\
\text { Case } 2 \\
\max .15 \mathrm{~min}\end{array}$} & \multirow[t]{2}{*}{ Tumor 1} & Tumor photographed? & & \\
\hline & & $\begin{array}{ll}\text { Correct localization? } & \text { Right: bronchus to basal part of lower lobe } \\
& \text { Right: lower lobe bronchus below segment } 6 \\
& \text { Right: bronchus to segment } 8,9 \text { and } 10\end{array}$ & & \\
\hline & \multirow[t]{2}{*}{ Tumor 2} & Tumor photographed? & & \\
\hline & & $\begin{array}{ll}\text { Correct localization? } & \text { Left: bronchus to apical/posterior part of upper lobe } \\
& \text { Left: bronchus to segment } 1 / 2\end{array}$ & & \\
\hline & \multicolumn{3}{|c|}{ Time $\rightarrow \rightarrow \rightarrow \rightarrow \rightarrow \rightarrow \rightarrow \rightarrow \rightarrow \rightarrow \rightarrow \rightarrow \rightarrow \rightarrow \rightarrow \rightarrow \rightarrow \rightarrow \rightarrow \rightarrow \rightarrow \rightarrow \rightarrow \rightarrow \rightarrow \rightarrow \rightarrow \rightarrow \rightarrow \rightarrow \rightarrow \rightarrow \rightarrow \rightarrow \rightarrow \rightarrow \rightarrow \rightarrow \rightarrow \rightarrow \rightarrow \rightarrow \rightarrow$} & \\
\hline \multirow{4}{*}{$\begin{array}{l}\text { Module 2: } \\
\text { Case } 1 \\
\operatorname{max.} 10 \mathrm{~min}\end{array}$} & \multicolumn{2}{|c|}{ At least one lavage performed in the middle lobe? } & & \\
\hline & \multicolumn{2}{|c|}{ Exactly three lavages performed in either lateral or medial part of middle lobe? } & & \\
\hline & \multicolumn{2}{|c|}{ Correct amount of suction? $\quad$ Between 30 and $45 \mathrm{~s}$ (both numbers included) } & & \\
\hline & \multicolumn{3}{|c|}{ Time $\rightarrow \rightarrow \rightarrow \rightarrow \rightarrow \rightarrow \rightarrow \rightarrow \rightarrow \rightarrow \rightarrow \rightarrow \rightarrow \rightarrow \rightarrow \rightarrow \rightarrow \rightarrow \rightarrow \rightarrow \rightarrow \rightarrow \rightarrow \rightarrow \rightarrow \rightarrow \rightarrow \rightarrow \rightarrow \rightarrow$} & \\
\hline \multirow{4}{*}{$\begin{array}{l}\text { Module 2: } \\
\text { Case } 2 \\
\max .10 \mathrm{~min}\end{array}$} & \multicolumn{2}{|c|}{ At least one lavage performed in the middle lobe? } & & \\
\hline & Exactly th & lavages performed in either lateral or medial part of middle lobe? & & \\
\hline & Correct a & ount of suction? $\quad$ Between 30 and $45 \mathrm{~s}$ (both numbers included) & & \\
\hline & Time $\rightarrow \rightarrow$ & $\Rightarrow \rightarrow \rightarrow \rightarrow \rightarrow \rightarrow \rightarrow \rightarrow \rightarrow \rightarrow \rightarrow \rightarrow \rightarrow \rightarrow \rightarrow \rightarrow \rightarrow \rightarrow \rightarrow \rightarrow \rightarrow \rightarrow \rightarrow \rightarrow \rightarrow \rightarrow \rightarrow \rightarrow \rightarrow \rightarrow \rightarrow \rightarrow \rightarrow \rightarrow \rightarrow$ & $\rightarrow \rightarrow \rightarrow \rightarrow$ & \\
\hline Biopsy tool & arm-up: $\mathbf{m}$ & dule 3 , case $1(\max .15 \mathrm{~min})$ & & \\
\hline Module 3: & Tumor pl & tographed? & & \\
\hline $\begin{array}{l}\text { Case } 3 \\
\max .20 \text { min }\end{array}$ & Correct lc & $\begin{array}{l}\text { Left: bronchus to anterior segment of upper lobe } \\
\text { Left: bronchus to segment } 3\end{array}$ & & \\
\hline & Correct la & age performed? & & \\
\hline & Correct $\mathrm{n}$ & dle biopsy performed? & & \\
\hline & Correct b & sh biopsy performed? & & \\
\hline & Exactly fo & forceps biopsies performed? & & \\
\hline & Time $\rightarrow \rightarrow$ & $\Rightarrow \rightarrow \rightarrow \rightarrow \rightarrow \rightarrow \rightarrow \rightarrow \rightarrow \rightarrow \rightarrow \rightarrow \rightarrow \rightarrow \rightarrow \rightarrow \rightarrow \rightarrow \rightarrow \rightarrow \rightarrow \rightarrow \rightarrow \rightarrow \rightarrow \rightarrow \rightarrow \rightarrow \rightarrow \rightarrow \rightarrow \rightarrow \rightarrow \rightarrow$ & $\rightarrow \rightarrow \rightarrow \rightarrow$ & \\
\hline Module 3: & Tumor pl & tographed? & & \\
\hline $\begin{array}{l}\text { Case } 4 \\
\max .20 \min \end{array}$ & Correct lo & $\begin{array}{l}\text { Left: bronchus to apical segment of lower lobe } \\
\text { Left: bronchus to segment } 6\end{array}$ & & \\
\hline & Correct la & age performed? & & \\
\hline & Correct $\mathrm{n}$ & dle biopsy performed? & & \\
\hline & Correct b & sh biopsy performed? & & \\
\hline & Exactly fo & forceps biopsies performed? & & \\
\hline & Time $\rightarrow \rightarrow$ & $\Rightarrow \rightarrow \rightarrow \rightarrow \rightarrow \rightarrow \rightarrow \rightarrow \rightarrow \rightarrow \rightarrow \rightarrow \rightarrow \rightarrow \rightarrow \rightarrow \rightarrow \rightarrow \rightarrow \rightarrow \rightarrow \rightarrow \rightarrow \rightarrow \rightarrow \rightarrow \rightarrow \rightarrow \rightarrow \rightarrow \rightarrow \rightarrow \rightarrow \rightarrow \rightarrow$ & $\rightarrow \rightarrow \rightarrow \rightarrow$ & \\
\hline & Total scor & $(\max .28)$ & & \\
\hline & Total tim & $\rightarrow \rightarrow \rightarrow \rightarrow \rightarrow \rightarrow \rightarrow \rightarrow \rightarrow \rightarrow \rightarrow \rightarrow \rightarrow \rightarrow \rightarrow \rightarrow \rightarrow \rightarrow \rightarrow \rightarrow \rightarrow \rightarrow \rightarrow \rightarrow \rightarrow \rightarrow \rightarrow \rightarrow \rightarrow \rightarrow \rightarrow \rightarrow \rightarrow \rightarrow \rightarrow$ & $\rightarrow \rightarrow \rightarrow \rightarrow$ & \\
\hline & Score per & linute & & \\
\hline
\end{tabular}


Table 2. Demographic data of the participants $(n=42)$

\begin{tabular}{|c|c|c|c|c|c|c|}
\hline Novices $(\mathrm{n}=14), 0$ bronchoscopies & 0 & 0 & 7 & 7 & $26(22-29)$ & $0(0-0)$ \\
\hline Trainees $(n=14), 10-100$ bronchoscopies & 9 & 5 & 7 & 7 & $39(31-49)$ & $50(15-90)$ \\
\hline Experts $(n=14), 500+$ bronchoscopies & 9 & 5 & 7 & 7 & $52(43-67)$ & $1,464(500-4,000)$ \\
\hline
\end{tabular}

Figures in parentheses are minimum to maximum values.

(to save test time, the participants were not asked to perform a complete bronchoscopy). Finally, the test included 2 cases where he or she had to find a tumor, state the localization and perform 1 lavage, 1 needle biopsy, 1 brush biopsy and 4 forceps biopsies of the tumor.

\section{Administration and Scoring}

The test was administered by L.K. who operated the simulator, handed over syringes and biopsy tools on request and intervened if the time limit was reached. During warm-up, he offered help and guidance with the simulator, the procedure and the anatomy, but during the test, there was no help or feedback apart from written instructions on the task and a map of the tracheobronchial tree. During the test, he also filled in the 28 items on the checklist. To make sure this was done in an objective and non-biased way, we recorded the bronchoscopies made by 10 of the participants ( 4 medical students, 3 trainees and 3 experts). We used a VGA splitter, a VGA to video converter and a Verbatim ${ }^{\mathrm{TM}}$ Mediastation HD DVR Network Multimedia Recorder 1 TB. When tumors were found, the exact words the participant used to describe their localizations were noted. The video recordings and descriptions of tumor localizations were reviewed by 2 blinded, independent raters (H.A. and C.v.B.) and their scoring was compared to the original score.

The time of each procedure was also noted on the checklist which allowed us to calculate an aggregate score, i.e. the bronchoscopy quality score, defined as the total score divided by the amount of time taken to perform the procedures. This was done to avoid the potential for bias if quality was assessed using only one measure - a quality bronchoscopy would be both fast and accurate [13].

\section{Statistical Analysis}

To assess the average correlation across all 3 raters (inter-rater reliability), Cronbach's $\alpha$ was calculated. One-way ANOVA was employed to establish construct validity, examining differences in performance between the 3 groups. A post-hoc test (StudentNewman-Keuls) was used to compare individual groups. Reliability was explored by the split-half approach using Pearson's correlation coefficient (r) and the Spearman-Brown (SB) 'prophecy' formula to estimate consistency of performance results across 3 pairs of similar procedures.
Statistical analysis was performed using a statistical software package (SPSS, version 15.0, SPSS Inc., Chicago, Ill., USA). Differences were considered to be statistically significant when the $p$ value was $<0.05$.

To examine the optimal balance between time consumption and precision of the test procedure, we used generalizability analyses as described by Brennan [17] in 2001. We adopted a 2-stage process: in the first stage, we obtained estimated variance components for a G study (single-facet, balanced design with test persons crossed with test items). The second stage involved using these estimated variance components in the context of a D study design to estimate generalizability coefficients - a reliability-like coefficient defined by Cronbach et al. [18] in 1972 - according to the number of tests included in the whole test procedure.

The $G$ and D studies were performed using the GENOVA software version 3.1 (Crick and Brennan 2001).

\section{Results}

The demographics of the participants are shown in table 2. The inter-rater reliability was high (Cronbach's $\alpha=$ 0.90). One-way ANOVA showed significant differences between groups for the parameters total time and checklist score $(p<0.001)$, whereas there were no significant differences for percent of bronchial segments entered and number of scope collisions with airway walls, i.e. the simulator metrics (table 3). Total time and checklist score were able to distinguish between experts/trainees and trainees/novices (Student-Newman-Keuls post-hoc test, $\mathrm{p}<0.05$ for all comparisons). Figure 1a shows probability density curves of the bronchoscopy quality scores for the 3 groups.

The performance of the persons in one half of the tests (checklist score) correlated with their performance in the other half $\left(\mathrm{r}=0.44, \mathrm{r}_{\mathrm{SB}}=0.61\right.$, correlation significant at the 0.01 level). Time spent on the first half and the second 
Table 3. Simulator metrics and performance data for novices, trainees and experts

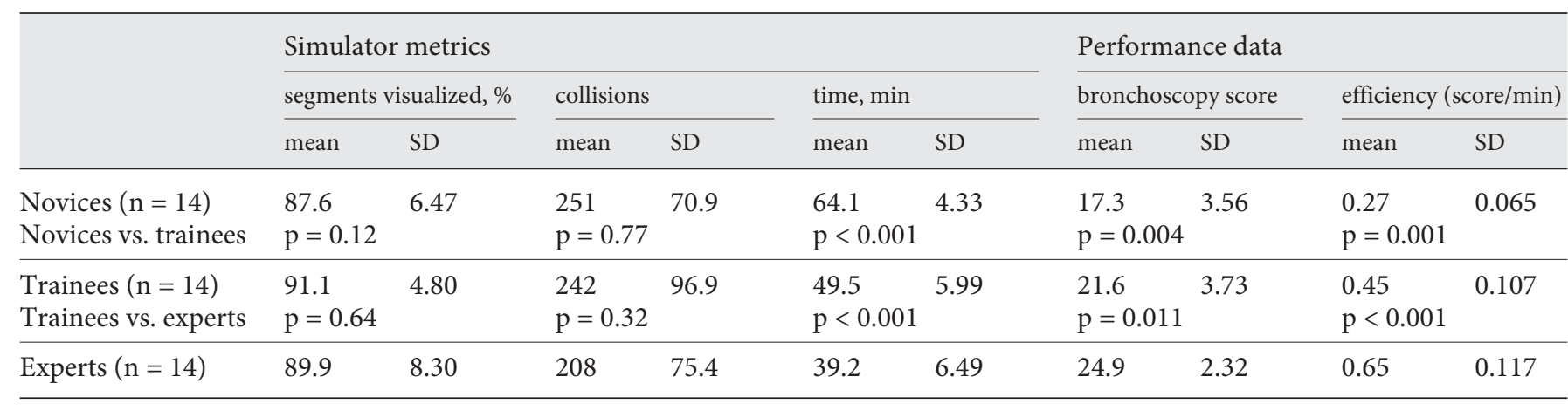
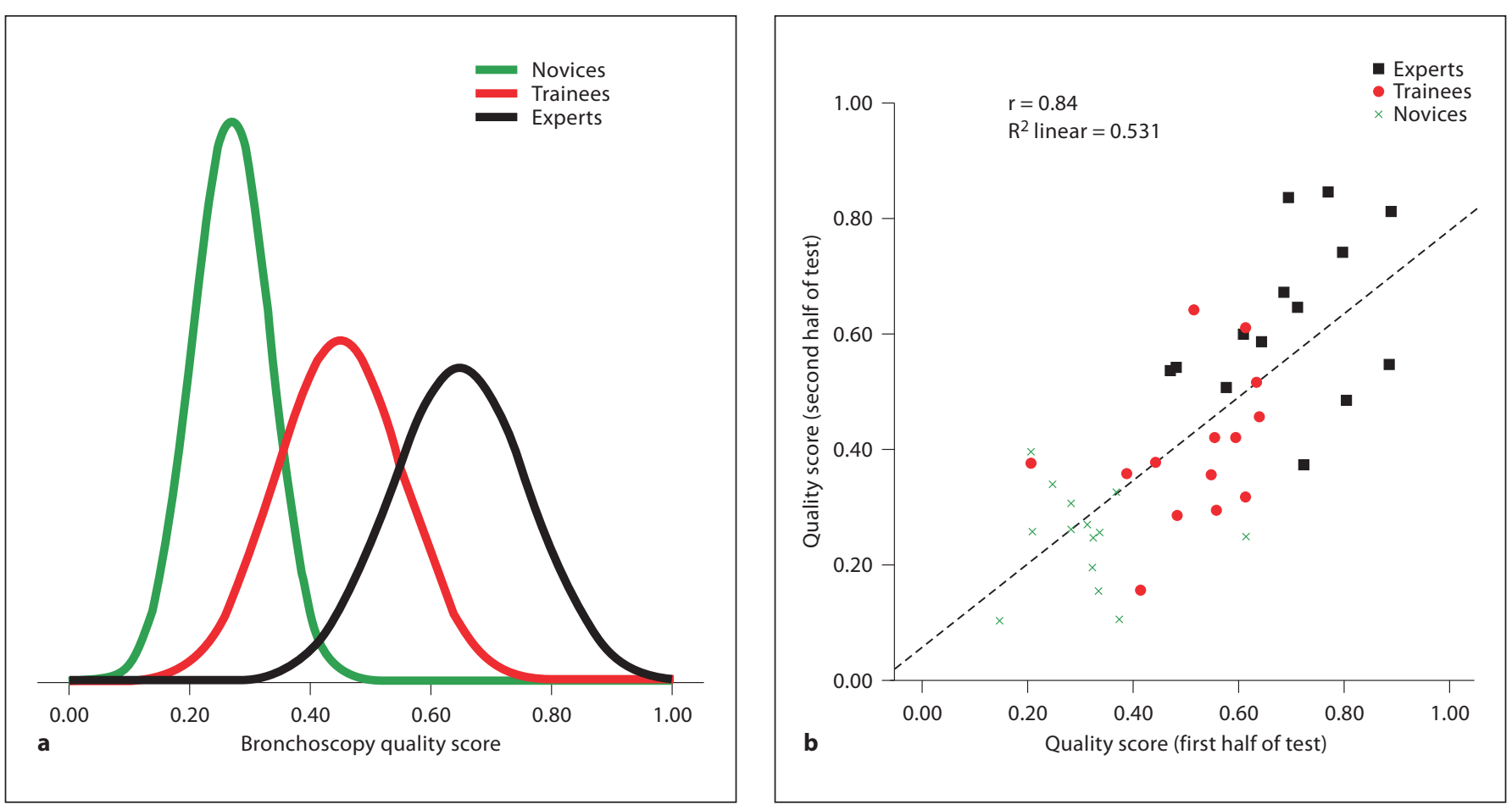

Fig. 1. a Construct validity. Probability density curves for bronchoscopy quality score (score/min). b Reliability. Performance: first half of test/second half of test.

half of the test also correlated $\left(\mathrm{r}=0.65, \mathrm{r}_{\mathrm{SB}}=0.79\right.$, correlation significant at the 0.01 level). The participants' splithalf correlation of the bronchoscopy quality score was even better; Pearson's correlation coefficient (2-tailed) was 0.73 uncorrected and 0.84 after correcting with the SB 'prophecy' formula (fig. 1b). However, the simulator metrics, percentage of segments visualized and number of collisions were not significantly correlated $(-0.13$ and 0.23 , respectively).

Figure 2 shows the effect on the generalizability coefficient according to the number of scenarios in the test program. The figure demonstrates that we need to test participants in 6 or more scenarios to secure a generalizability coefficient $>0.80$. 


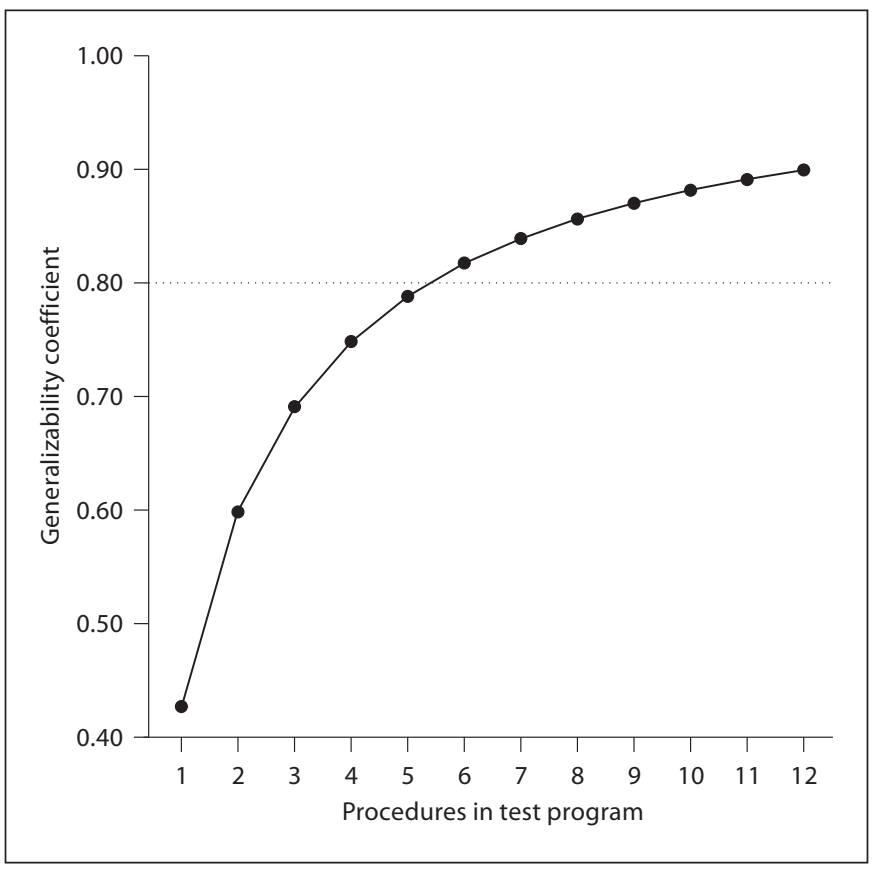

Fig. 2. Size of test program. Generalizability coefficient depending on number of exercises.

\section{Discussion}

To our knowledge, this article is the first to present a reliable and valid assessment procedure regarding bronchoscopy performance that can discriminate between intermediate bronchoscopists and experts. Furthermore, it is the first assessment tool that can be scored in a totally objective way, using blinded raters. Validity was supported by our findings that experts scored significantly better than trainees who in turn scored significantly better than novices (fig. 1a). Reliability was demonstrated by high split-half correlations (fig. 1b) and a high generalizability coefficient when assessing across 6 scenarios (fig. 2).

Using video recordings to assess procedures, we can avoid all of the potential pitfalls of human relations: subjectivity, false impressions, ageism, racism, sexism, rumor, grudge and misinterpretation [19]. The raters do not need to be present when the procedures are performed, but post-procedure assessment is time-consuming. Furthermore, the blinded recordings do not allow the raters to observe other important aspects of a bronchoscopy procedure, i.e. the interactions with the patient and the assisting staff or correct handling of the scope that prevents costly damage [20]. This must be done unblinded using direct observation. To minimize subjectivity, we used a checklist instead of a rating scale - because rating scales require the exercise of judgment, they are inherently more subjective than checklists [21]. Indeed, our 3 raters (1 non-blinded, 2 blinded) scored the checklist in a very uniform way.

We believe that 4 factors are crucial to the reliability and validity of our instrument: a standardized setting, a scoring system with clinical relevance, the testing with tasks of different difficulty levels, and multiple test items.

The virtual reality bronchoscopy simulator provides a standardized, relatively stress-free environment ideally suitable for objective assessment of trainees.

Our study showed that the novel measurement instrument sufficiently discriminated the performance between operator groups. However, similar to other studies, we found that the simulator metrics did not possess this ability [13]. One example is the data of 'percentage of segments visualized' where novice scores were at the level of experts. A reason for this could be that several novices got a high score by moving around the bronchial tree like a mouse in a maze - entering many or all segments without knowing exactly where they were. All novices can enter all segments after very little practice [22], which means that the task is too easy to use as a performance criterion. It is highly probable that the addition of clinically relevant parameters in our checklist score overcomes this problem.

Moreover, the inclusion of tasks with increasing difficulty in the entire assessment approach helps to avoid a ceiling effect, as one of the trademarks of experts is the ability to transfer skills to a new situation [16].

Finally, the sufficient validity and reliability of our instrument probably relates to our decision to increase the number of test scenarios, and hence test items, in our assessment compared to earlier studies rating 1 or 2 bronchoscopies $[13,15]$. The simplest way to improve reliability of a measurement instrument is to increase the number of items, because the true variance will increase by the square of the number of items, whereas the error variance will increase only by the number of items [23]. The downside of increasing the number of items in a test is that it will take longer to perform. We found a median test time including a 'warm-up' of 59 min (range 42-80) for experts and trainees. The novices took more time to instruct, and test, but usually, there is no need to test the skills of people with no prior knowledge of bronchoscopy. The checklist was filled in during the test, and calculation of the bronchoscopy quality score took only a few minutes. Our D study (fig. 2) showed that the reliability of the test program would drop considerably if we chose to save test time by reducing the number of procedures. If we 
chose to double the number of procedures in the test, the generalizability coefficient would improve (from 0.82 to 0.90 ). However, as a coefficient $>0.8$ is generally considered acceptable and a test time of approximately $1 \mathrm{~h}$ should be feasible, we recommend the assessment program in its original form (6 procedures).

The new test procedure has other limitations apart from being moderately time-consuming: the necessary virtual reality bronchoscopy simulator is still sold at a prohibitive price (USD $>120,000$ ) [24]. Additionally, the test cannot be administered to the same person several times without a significant learning-by-testing effect because the number of available patient cases in the software is rather limited. This has become a problem in Denmark after training on the bronchoscopy simulator has become mandatory for all physicians specializing in thoracic surgery and pulmonary medicine. However, as the test procedure does not use data specific to the simulator, it is possible to perform in other settings, i.e. a low-fidelity mannequin, with only minor adjustments. Actually, a recent study on training in conventional transbronchial needle aspiration found that a cheap lowfidelity model was superior to an expensive high-fidelity platform [25]. We recommend the use of our validated test to perform criterion validation of future assessment tools, whether they are based on patients, mannequins or new simulators $[26,27]$.

Whether our assessment instrument and procedure will prove valid and reliable when applied to performing real bronchoscopies remains to be shown. A recent study about performance of bronchoscopies in real patients successfully used an assessment method that was validated on a simulator to prove the effect of a new simulationbased training program. However, whether performance in the simulation setting predicts clinical performance is still a subject for future research. Finally, if our assessment approach should be used for certification of operators, there is a need for setting standards of pass/fail criteria.

Although much more research is needed, this study is an important first step in developing a valid and reliable assessment procedure. Using virtual reality simulation for this purpose could be valuable for the standardization of the assessment without jeopardizing patient safety or interfering with clinical service demands and time constraints.

\section{Conclusion}

The new simulator-based bronchoscopy assessment procedure is an easy-to-use and relatively fast test that can be used to precisely assess bronchoscopy skills. The test is valid and reliable, and the length of the test is appropriate. The novel assessment approach should be used to provide important feedback to bronchoscopy trainees and to measure the effect of new teaching curricula, i.e. involving bronchoscopy simulators. Also, it might prove valuable to decide when trainees have reached sufficient basic competency.

\section{Acknowledgments}

We thank Dr. Nils Milman for his aid in the construction of the assessment tool. This work was in part supported by the Research Foundation of the Heart Center, Rigshospitalet, Copenhagen, Denmark.

\section{Financial Disclosure and Conflicts of Interest}

Dr. Konge, Dr. Arendrup, Professor Buchwald and Professor Ringsted declare no conflicts of interest or financial disclosure.

\section{References}

1 Ernst A, Silvestri GA, Johnstone D: Interventional pulmonary procedures: Guidelines from the American College of Chest Physicians. Chest 2003;123:1693-1717.

-2 Ouellette DR: The safety of bronchoscopy in a pulmonary fellowship program. Chest 2006;130:1185-1190.

3 Knight RK, Clarke SW: An analysis of the first 300 fibreoptic bronchoscopies at the Brompton Hospital. Br J Dis Chest 1979;73: 113-120.
-4 Hsu LH, Liu CC, Ko JS: Education and experience improve the performance of transbronchial needle aspiration: a learning curve at a cancer center. Chest 2004;125:532-540.

$\checkmark 5$ British Thoracic Society guidelines on diagnostic flexible bronchoscopy. Thorax 2001; 56(suppl 1):i1-i21.

6 Prakash UB, Offord KP, Stubbs SE: Bronchoscopy in North America: the ACCP survey. Chest 1991;100:1668-1675.
-7 Pastis NJ, Nietert PJ, Silvestri GA: Variation in training for interventional pulmonary procedures among US pulmonary/critical care fellowships: a survey of fellowship directors. Chest 2005;127:1614-1621.

$>8$ Moorthy K, Munz Y, Sarker SK, et al: Objective assessment of technical skills in surgery. BMJ 2003;327:1032-1037.

9 Reznick RK, MacRae H: Teaching surgical skills - changes in the wind. N Engl J Med 2006;355:2664-2669. 
10 Crawford SW, Colt HG: Virtual reality and written assessments are of potential value to determine knowledge and skill in flexible bronchoscopy. Respiration 2004;71:269275.

11 Quadrelli S, Davoudi M, Galindez F, et al: Reliability of a 25 -item low-stakes multiplechoice assessment of bronchoscopic knowledge. Chest 2009;135:315-321.

12 Blum MG, Powers TW, Sundaresan S: Bronchoscopy simulator effectively prepares junior residents to competently perform basic clinical bronchoscopy. Ann Thorac Surg 2004;78:287-291.

13 Ost D, DeRosiers A, Britt EJ, et al: Assessment of a bronchoscopy simulator. Am J Respir Crit Care Med 2001;164:2248-2255.

14 Moorthy K, Smith S, Brown T, et al: Evaluation of virtual reality bronchoscopy as a learning and assessment tool. Respiration 2003;70:195-199.
15 Davoudi M, Osann K, Colt HG: Validation of two instruments to assess technical bronchoscopic skill using virtual reality simulation. Respiration 2008;76:92-101.

16 Magill RA: Defining and Assessing Learning. Motor Learning and Control. New York, McGraw-Hill, 2007, pp 246-262.

17 Brennan RL: Generalizability Theory, ed 1. New York, Springer, 2001.

18 Cronbach LJ, Gleser GC, Nanda H, et al: The Dependability of Behavioral Measurements, ed 1. New York, John Wiley and Sons, 1972.

19 McGaghie WC, Butter J, Kaye M: Observational assessment; in Downing SM, Yudkowsky R (eds): Assessment in Health Professions Education. New York, Routledge, 2009, pp 185-215.

20 Rozman A, Duh S, Petrinec-Primozic M, et al: Flexible bronchoscope damage and repair costs in a bronchoscopy teaching unit. Respiration 2009;77:325-330.

21 Yudkowsky R: Performance tests; in Downing SM, Yudkowsky R (eds): Assessment in Health Professions Education. New York, Routledge, 2009, pp 119-148.
22 Colt HG, Crawford SW, Galbraith O III: Virtual reality bronchoscopy simulation: a revolution in procedural training. Chest 2001; 120:1333-1339.

23 Streiner DL, Norman GR: Health Measurement Scales - A Practical Guide to Their Development and Use. Oxford, Oxford University Press, 2008, p 170.

24 Davoudi M, Colt HG: Bronchoscopy simulation: a brief review. Adv Health Sci Educ Theory Pract 2009;14:287-296.

25 Davoudi M, Wahidi MM, Zamanian RN, et al: Comparative effectiveness of low- and high-fidelity bronchoscopy simulation for training in conventional transbronchial needle aspiration and user preferences. Respiration 2010;80:327-334.

26 GI-BRONCH Mentor. 2010. http://www. simbionix.com/BRONCH/BRONCH.html.

27 Airway Skills Bronchoscopy Simulator. 2010. http://www.airwayskills.com/files/ downloads/airwayskills_bronchoscopy_ simulator.pdf. 